\title{
Assessment of the lactate biosensor methodology
}

\author{
B. Detry, W. Nullens, M.L. Cao, A. Frans, A. Robert, T. Clerbaux
}

\begin{abstract}
Assessment of the lactate biosensor methodology. B. Detry, W. Nullens, M.L. Cao, A. Frans, A. Robert, T. Clerbaux. (CERS Journals Ltd 1998.

ABSTRACT: The rapid determination of lactate level is useful for clinical emergencies, as in the case of shock conditions or during surgical operations, as well as in numerous cases of respiratory failure, in cardiac or paediatric pathology and during exercise tests. Moreover, it is of prognostic significance in critically ill patients. Photometric methods are slow and, even when performed in good conditions, will give results only $30 \mathrm{~min}$ after blood collection, during which time the clinical condition of the patient may change. In this study, we have assessed the lactate biosensor, a method that yields lactate measurements in less than 1 min with only $100 \mu \mathrm{L}$ of biological fluid.

In order to test the validity of this method, we performed comparisons between the Sigma classical enzymatic reference method and two commercially available biosensors: the Ciba-Corning biosensor 865 and the Yellow Springs lactate biosensor. Lactate measurements were performed in heparinized arterial blood samples without antiglycolitic agent $(n=71)$. In order to cover a wide range of lactate levels, samples came from patients admitted to the intensive care unit for severe conditions and patients addressed for bicycle exercise testing. Each whole blood sample was processed in duplicate by both biosensors. For plasma measurement, subsamples of whole blood were centrifuged and the resulting plasma were processed by the biosensors and the Sigma method. Two parameters that can potentially influence lactate measurement were also investigated: haematocrit and total protein levels.

The data showed that measurements performed on plasma are satisfactory for both biosensors. For whole blood, the Ciba-Corning device gives accurate results but the Yellow Springs apparatus seriously underestimates lactate levels. This underestimation is strongly influenced by the haematocrit level, so that a correction factor can be calculated (based on the haemoglobin level), which allows accurate "corrected" results to be obtained for whole blood with the Yellow Springs analyser.

Eur Respir J 1998; 11: 183-187.
\end{abstract}

Cliniques Universitaires Saint-Luc, Université Catholique de Louvain, Brussels, Belgium.

Correspondence: T. Clerbaux

Cliniques Universitaires Saint-Luc, UCL 10-2832

10 Av. Hippocrate

1200 Brussels

Belgium

Fax: 003227645536

Keywords: Lactate biosensor methodology

Received: January 141997

Accepted after revision July 311997

This study was partly supported by a grant from Ciba-Corning Diagnostic Corporation, Medfield, MA, USA, (now Chiron Diagnostics).
Patients with failure of vital functions are usually cared for in high technology labour-intensive specialized units, where the affected vital functions can be assisted or supplemented for variable periods of time during spontaneous or medically driven recovery. The main purpose of support therapy in intensive care units is to grant a sufficient physiological level of cellular metabolism in order to avoid cellular damage.

One of the best indicators of cellular integrity in critically ill patients is the measurement of blood lactate levels. Although the actual blood lactate value depends not only on lactate production, but also on lactate metabolism and, thus, is not a straightforward indicator of overall cell function in the body, it has been shown to have prognostic value in critically ill patients [1]. Moreover, lactate levels may be useful in the short-term follow-up of the evolution of this kind of patient and thus, can be used to guide medical interventions.

Blood lactate levels measurements are performed using a variety of methods, including enzymatic and biosensorbased techniques. Lactate can be measured either in whole blood or in plasma samples, and its value can be influ- enced by several factors including the sampling methodology (especially dilution of the sample), the measurement method itself, haemoglobin levels and protein levels in the sample. In the present study, we evaluated a new biosensor-based method (Ciba-Corning 865; Ciba-Corning Diagnostic Corporation, Medfield, MA, USA) and compared it with an older biosensor-based technique (YSI 2300; Yellow Springs Instrument Co., Yellow Springs, OH, USA) and with the classic enzymatic reference method (Sigma Diagnostics, St Louis, MO, USA).

\section{Materials and methods}

Lactate measurements were performed in arterial blood samples $(\mathrm{n}=71)$ obtained from 40 patients. Routine measurements of lactate were performed using the YSI 2300 analyser. Samples were not chosen randomly, but were selected in order to cover a wide range of lactate levels. Samples came from two types of patients: those admitted to the intensive care unit for severe conditions; and those attending the pneumology department for blood gas measurements during bicycle exercise testing. 
All lactate measurements were performed immediately after blood sampling in order to avoid an increase in lactate concentration. For each sample, $10 \mathrm{~mL}$ of heparinized arterial blood, without antiglycolitic agent, was processed in duplicate as follows: 1) for whole blood lactate measurement, whole blood was injected simultaneously into both Ciba-Corning 865 and YSI 2300 analysers; and 2) for plasma lactate measurement a subsample of whole blood was centrifuged to precipitate the red blood cells and the resulting plasma was injected simultaneously into the Ciba-Corning and in the YSI 2300 analysers. Furthermore, $1 \mathrm{~mL}$ of plasma was diluted (1:3) in $8 \%$ perchloric acid in order to obtain the classical deproteinized sample used for lactate measurement by the enzymatic method of the Sigma Company (procedure No. 826-UV).

Two parameters that can potentially influence the lactate measurement were also investigated: haematocrit and total protein levels. Haematocrit values were obtained by microcentrifugation with Hettich Universal 30 RF centrifuge (Hettich Tuttlingen, Germany) at 15,000 revolutions per minute (rpm) for $3 \mathrm{~min}$. Total protein level was measured in plasma samples by the Sigma method (procedure No. 541) using the classical colorimetric Biuret method.

Whole blood lactate levels for another set of 350 arterial blood samples were measured by the both YSI and Ciba-Corning analysers. For each of these 350 samples, the haemoglobin level was measured by the OSM3 analyser (Radiometer, Copenhagen, Denmark). This measurement allowed calculation of the haematocrit of the sample using the equation: haematocrit $=2.941 \times$ haemoglobin [2]. The validity of this equation was tested in 59 samples, comparing the measured value of haematocrit obtained by microcentrifugation and the calculated value using a paired t-test.

\section{Technical details}

The 2300 YSI and the Ciba-Corning 865 lactate analysers are based on an electrochemical sensor using the reaction, catalysed by lactate oxidase (LOD), that transforms lactic acid into pyruvic acid and hydrogen peroxide $\left(\mathrm{H}_{2} \mathrm{O}_{2}\right)$. The produced $\mathrm{H}_{2} \mathrm{O}_{2}$ is oxidized to produce electrons that create an electrical current directly proportional to the lactate concentration in the sample. In both detectors, the sample is separated from the electrode by a semipermeable membrane allowing only water and metabolites to pass. For the YSI analyser, the LOD is immobilized on a second membrane, a third membrane readily passes $\mathrm{H}_{2} \mathrm{O}_{2}$ (excluding compounds with molecular weight $>200$ ). The lactate measurement requires an isotonic 1:25 dilution of the sample. The Ciba-Corning lactate biosensor includes a measuring electrode that contains LOD in a binder and uses an interference electrode; the measurement is made on an undiluted sample.

In the enzymatic Sigma lactate procedure, the following reaction catalysed by lactate dehydrogenase $(\mathrm{LDH})$ is used:

$$
\text { Lactate }+\mathrm{NAD}^{+}=\text {Pyruvate }+\mathrm{NADH}
$$

where $\mathrm{NAD}^{+}$is $\beta$-nicotinamide adenine dinucleotide and $\mathrm{NADH}$ is $\beta$-nicotinamide adenine dinucleotide, reduced form.
This reaction is carried to the right with excess $\mathrm{NAD}^{+}$. To force the reaction to completion in this direction, the pyruvate produced is trapped with hydrazine. The increased absorbance at $340 \mathrm{~nm}$ due to NADH is directly proportional to the lactate level of the sample. The procedure is described for whole blood, i.e. to measure the plasma lactate and the red blood cell lactate content, but we used the same procedure on plasma samples in order to best approach the measurements performed with the biosensor devices. The Sigma plasma lactate procedure was considered as the reference method.

\section{Data analysis}

Because of a proportional relationship between measured standard deviations, data were analysed on a logarithmic scale. Moreover, as the studied methods gave closely related measurements, the usual plot, method one versus method two on a X-Y plot, is inefficient because all the points tend to be clustered along the lines of identity. Therefore, the difference was plotted against the average of the measurements by the two methods as described by Bland and Altman [3, 4]. On a logarithmic scale, such a plot becomes $\log$ (test) - $\log$ (reference) as a function of the average of $\log$. The antilog of data transforms the ordinate into the geometric difference (test/reference) and abscissa into geometric mean of test and reference. The $95 \%$ confidence interval $(95 \% \mathrm{CI})$ on geometric difference was also calculated and reported on the graph.

For each method, the intraclass correlation coefficient (ICC) was estimated from the two measurements using a one-way analysis of variance (ANOVA) with random effect. Each tested method was compared with the reference method using a one-way ANOVA with fixed effect, and the reliability coefficient was estimated.

The effect of the haematocrit level on the difference of measurement between whole blood YSI and reference method was assessed by linear regression. The regression equation allowed the calculation of a predicted error of the YSI analyser due to haematocrit. Therefore, the ratio adjusted for haematocrit level was plotted against reference method and the intermethod reliability coefficient was estimated for this "corrected" whole blood YSI lactate level.

\section{Results}

For each method, the ICC was greater than 0.997. This result justifies the use of the average of the two measurements as one variable for the rest of the calculations.

The differences between the tested method and the Sigma procedure (reference) were plotted as a function of the average of tested and reference method. This plot showed an increase in the scatter of the differences and a tendency for the mean difference to fall with increasing magnitude of measurement. As stated above, a logarithmic transformation was applied to data in order to resolve this problem. The plot became geometric difference between tested and reference methods as a function of geometric mean of both methods. The tested methods were: plasma YSI lactate (fig. 1a); whole blood Corning 
lactate (fig. 1b); plasma Corning lactate (fig. 1c); and whole blood YSI lactate (fig. 2a). The $95 \%$ CI of geometric difference was reported on graphs and in table 1 . The graphs showed that all tested methods gave a mean ratio close to 1 (fig. 1) except the whole blood YSI (fig. 2a). Moreover, the $95 \%$ CI was close to 0.40 for all methods excepted for plasma lactate YSI. The latter method was the one that had the best agreement with the reference method.

The estimation of the interclass relability coefficient (R) led to the same interpretation (table 2), with the higher $\mathrm{R}$ value for plasma lactate YSI and the worst for whole blood YSI.
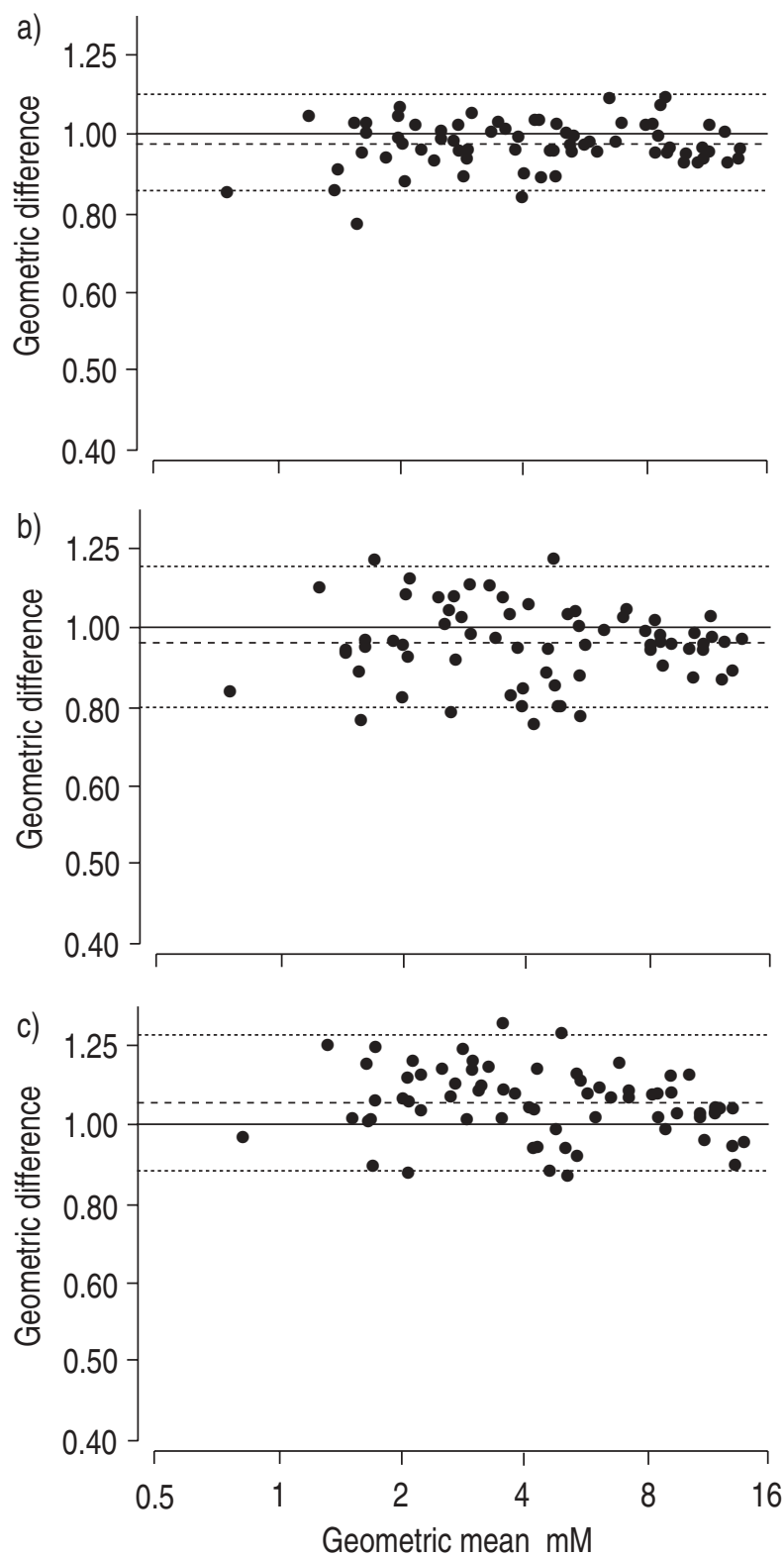

Fig. 1. - Plot of the geometric difference between the tested method and the Sigma procedure as a function of the geometric mean of tested and reference method. The tested methods were: a) plasma lactate from the YSI 2300; b) whole blood lactate from the Ciba-Corning analyser; and c) plasma lactate from the Ciba-Corning analyser. Geometric difference: analyser value/reference; Geometric mean: Đanalyser value $\times$ reference. - - - - : mean of geometric difference; .......... : $95 \%$ confidence interval of geometric difference
Linear regression of $\log$ difference between methods versus the haematocrit and total protein levels were calculated in order to assess the effect of these parameters on the difference between measurements. Total protein showed no significant relationship with log difference of lactate measurement but the haematocrit level was significantly correlated with the difference between log whole blood
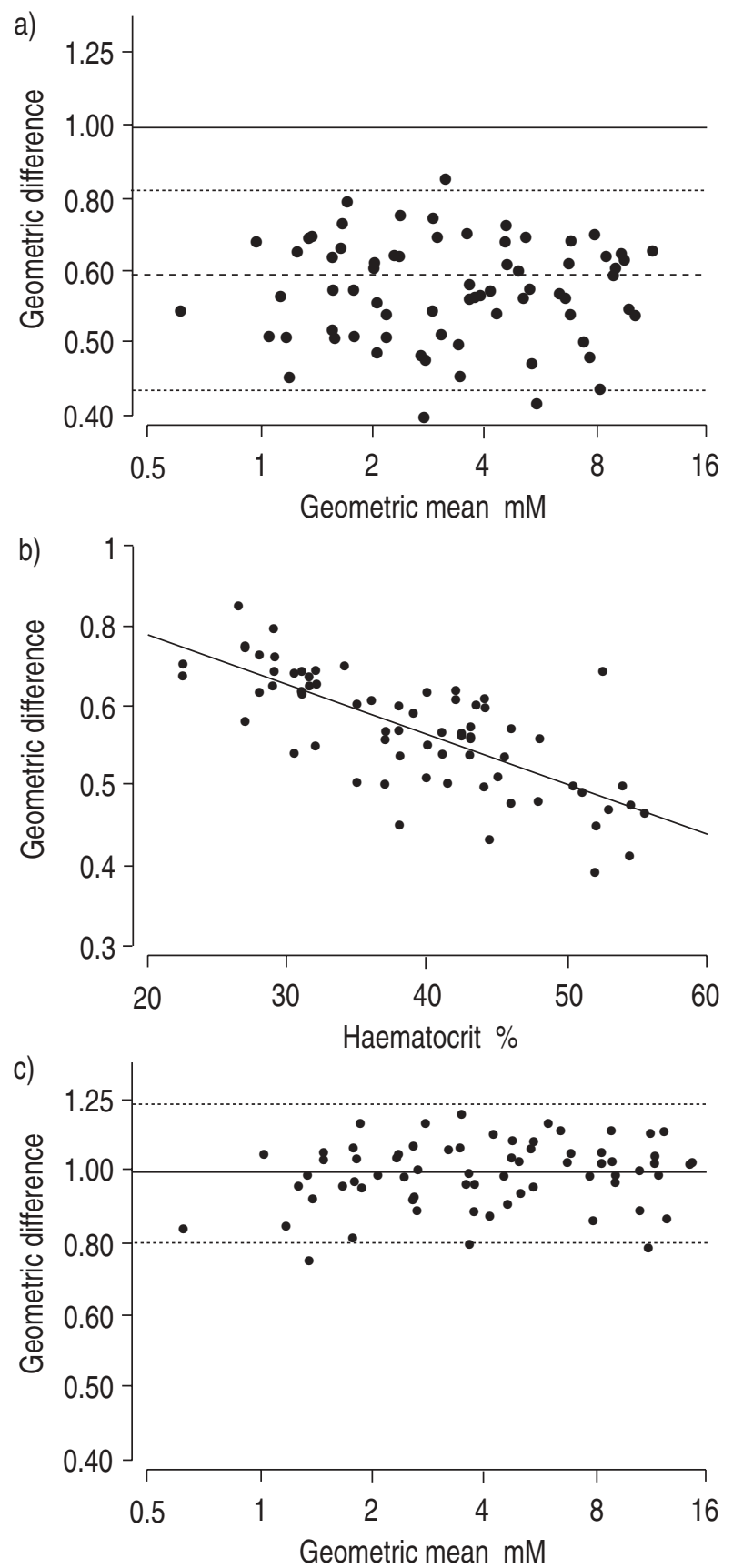

Fig. 2. - a) Plot of the geometric difference between the whole blood lactate measured with the YSI analyser and the reference method as a function of geometric mean of whole blood lactate from the YSI analyser and reference methods; b) plot of the geometric difference between the whole blood lactate from the YSI analyser and the reference method as a function of the haematocrit level of the sample; and c) plot of the ratio adjusted for haematocrit level as a function of the geometric mean of tested and reference methods. Adjustment for haematocrit: (whole

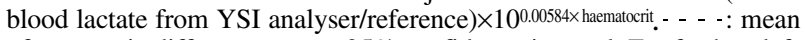
of geometric difference; …...95\% confidence interval. For further definitions, see legend to figure 1. 
Table 1. - 95\% confidence intervals (95\% Cls) of geometric difference between tested and reference methods

\begin{tabular}{lcc}
\hline \multicolumn{1}{c}{ Tested method } & Mean & $95 \%$ CI \\
\hline Whole blood YSI & 0.5930 & $0.4302-0.8174$ \\
Plasma YSI & 0.9743 & $0.8512-1.1150$ \\
Whole blood Corning & 0.9567 & $0.7709-1.1873$ \\
Plasma Corning & 1.0565 & $0.8775-1.2718$ \\
\hline
\end{tabular}

YSI: YSI 2300 lactate biosensor (Yellow Springs Instrument Co., Yellow Springs, OH, USA); Corning: Ciba-Corning 865 lactate biosensor (Ciba-Corning Diagnostic Corporation, Medfield, MA, USA).

Table 2. - One-way ANOVA results: calculated F-values and estimated interclass reliability coefficients $(R)$

\begin{tabular}{lccc}
\hline \multicolumn{1}{c}{ Tested method } & $\begin{array}{c}\text { Between } \\
\text { samples } \\
\mathrm{F}\end{array}$ & $\begin{array}{c}\text { Between } \\
\text { methods } \\
\mathrm{F}\end{array}$ & $\mathrm{R}$ \\
\hline Whole blood YSI & 73.80 & 722.88 & 0.8568 \\
Plasma YSI & 425.60 & 10.19 & 0.9950 \\
Whole blood Corning & 161.65 & 11.48 & 0.9868 \\
Plasma Corning & 215.50 & 23.84 & 0.9893 \\
\hline
\end{tabular}

ANOVA: analysis of variance. For further definitions see legend to table 1.

YSI and log reference (fig. 2b) according to the following equation:

$$
\begin{gathered}
\log \text { whole blood YSI - } \log \text { reference }=-5.84 \times 10^{-3} \times \\
\text { haematocrit } \mathrm{r}^{2}=0.54 .
\end{gathered}
$$

This result allows calculation of a predicted error for the whole blood YSI measurement taking into account the haematocrit level of the sample:

$$
\begin{gathered}
\log \text { whole blood } \mathrm{YSI}+5.84 \times 10^{-3} \times \text { haematocrit }= \\
\log \text { reference }
\end{gathered}
$$

and, taking the antilog:

$$
\text { whole blood YSI } \times 10^{0.00584 \text { \hamatocrit }=\text { reference. }}
$$

Therefore, we plotted the ratio adjusted for haematocrit

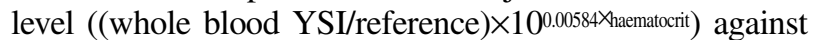
the geometric mean of corrected whole blood YSI and reference methods (fig. 2c). This latter plot showed that after correction, the obtained variation interval was comparable with that of the other tested methods.

To verify the accuracy of this correction for the haematocrit, we applied our correction factor to an independent set of data $(n=350)$, in which the whole blood YSI and whole blood Corning lactate levels and the haemoglobin had been measured for each sample. Whole blood Corning lactate was considered as equal to the reference for this comparison. The validity of the equation [2] that estimates the haematocrit value knowing the haemoglobin level (haematocrit $=2.941 \times$ haemoglobin) was verified: the measured value (microcentrifugation) was compared to the calculated one for a subset of 59 samples by a paired t-test. This test leads to the conclusion that there was no significant difference between the two haematocrit estimations $(\mathrm{t}=0.27 ; \mathrm{p}=0.79)$.
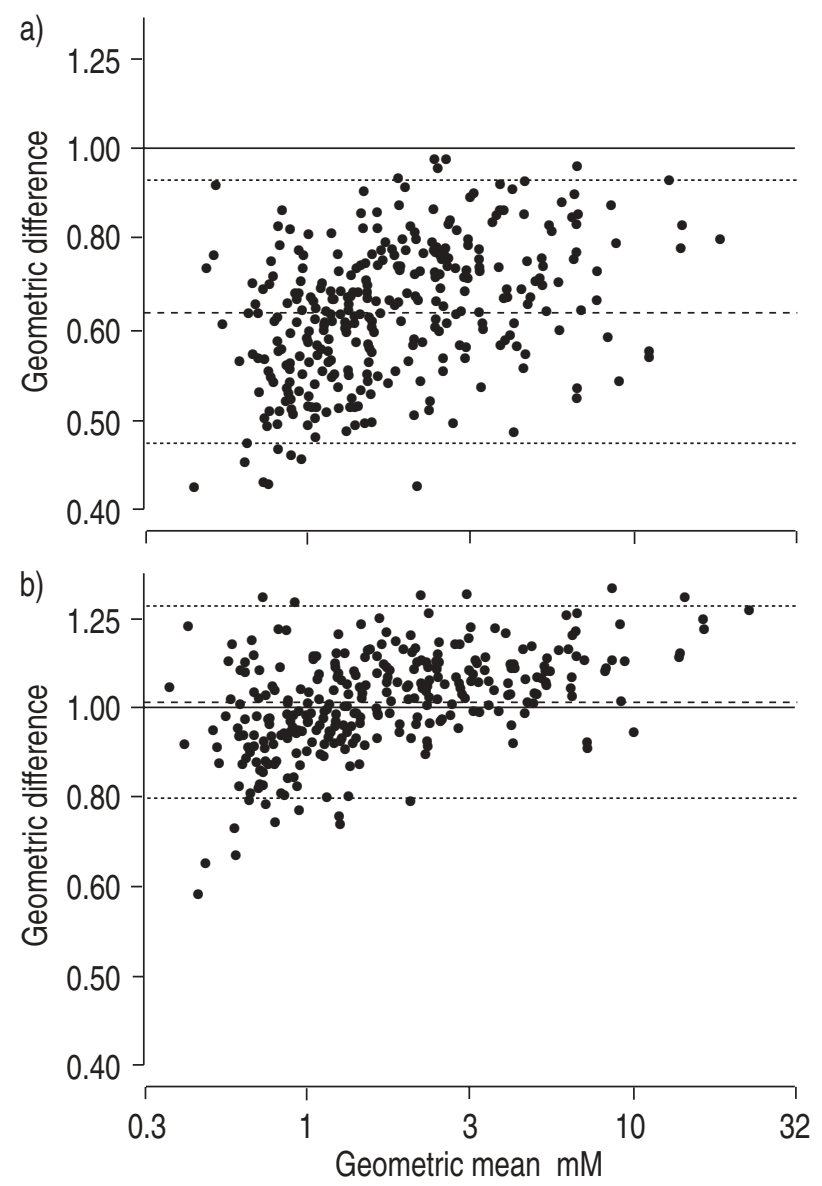

Fig. 3. - a) Plot of the geometric difference between the whole blood lactate measured with the YSI analyser and whole blood lactate measured by the Ciba-Corning analyser as a function of the geometric mean of the two methods; and b) plot of the ratio adjusted for haematocrit level as a function of the geometric mean of whole blood lactate from the YSI analyser corrected for haematocrit and whole blood lactate from the Ciba-Corning analyser. Geometric difference: whole blood lactate from YSI/whole blood lactate from Ciba-Corning; Geometric mean: $\mathrm{(whole}$ blood lactate from YSI $\times$ whole blood lactate from Ciba-Corning); adjustment for haematocrit: (whole blood lactate from YSI whole blood lactate

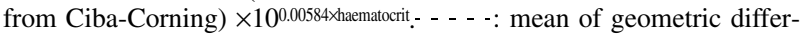
ence; $\cdots \cdots \cdots . . . .95 \%$ confidence interval.

The plot of geometric difference (whole blood YSI/ whole blood Corning) against geometric mean (fig. 3a) showed results comparable with those obtained from the first set of 71 samples (mean $=0.66$, lower limit $=0.47$, higher limit=0.93). When the correction factor for haematocrit was applied, the mean became 1.02 and the $95 \%$ CI limits 0.79 and 1.31 (fig. 3b).

\section{Discussion}

In this study, we compared blood lactate measurements performed on whole blood and plasma samples using two commercially available biosensor devices with the reference chemical method. The data show that measurements performed on plasma are satisfactory both for the YSI and Corning analysers. By contrast, for measurements performed on whole blood, the Corning device gives quite accurate results but the YSI analyser seriously underestimates lactate levels. This underestimation is strongly influenced by the haematocrit level of the sample, so that a 
correction factor can be calculated (based on the haemoglobin level of the sample), which allows the obtention of accurate "corrected" results for whole blood with the YSI analyser.

The main advantage of the Ciba-Corning device is that whole blood samples can be used to obtain accurate lactate measurements. The YSI apparatus does not give satisfactory results on whole blood. Therefore, one of two options are available. Either the whole blood needs to be centrifuged in order to separate the plasma in which the measurements obtained with the YSI analyser are accurate, or one has to measure haemoglobin independently in order to apply a correction factor to the measurement obtained on the whole blood. This has the disadvantage of giving a result that is not a measurement but a calculation. Although our own data show that the correction formula fully compensates for the underestimation of the YSI method, results based on calculations should not be favoured over data obtained directly from measurements. Moreover, the independent measurement of haemoglobin or the centrifugation needed for the YSI method makes this methodology longer to perform and has the disadvantage of introducing an independent measurement with its own accuracy and reproducibility problems. YSI sells tubes where whole blood samples can be immediately placed, for cell lysis before analysis, in order to minimize the problem of underestimation of the YSI 2300 when measuring whole blood lactate. This procedure leads to an underestimation of $\pm 20 \%$ of the plasma lactate concentration [5] and, therefore, the error is partially corrected. However, there is a significant disadvantage with this technique: the measured value is the total lactate in the total blood volume independent of the partitioning function provided by the red blood cells. However, only the extracellular, plasma lactate is physiologically dynamic and relevant and the main advantage of biosensors is their ability to measure the plasma concentration of electrolytes in a whole blood sample.

In conclusion, both the YSI and Ciba-Corning devices based on biosensors membranes can be used to obtain accurate data for blood lactate levels, both in whole blood and plasma, in a rapid and reproducible way. However, correction and/or supplementary manipulations are needed to use the YSI method on whole blood. The CibaCorning analyser thus appears simpler and/or faster than the YSI one.

Acknowledgements: The authors gratefully acknowledge the critical reading of the manuscript by $\mathrm{D}$. Rodenstein, as well as his constructive suggestions.

\section{References}

1. Alduen J, Bernstein WK, Khastgir T, et al. The use and clinical importance of a substrate-specific electrode for rapid determination of blood lactate concentrations. JAMA 1994; 272: 1678-1685.

2. Geigy Scientific Tables, 6th edition, 1963 p. 560.

3. Bland JM, Altman DG. Comparing methods of measurements: why plotting difference against standard method is misleading. Lancet 1995; 346: 1085-1087.

4. Bland JM, Altman DG. Statistical methods for assessing agreement between two methods of clinical measurement. Lancet 1986; i: 307-310.

5. Foxdal P, Bergqvist Y, Eckerbom S, Sandhagen B. Improving lactate analysis with the YSI 2300 GL: hemolysing blood samples makes results comparable with those for deproteinized whole blood. Clin Chem 1992; 38: 2110 2114. 